\title{
Low-Cost Dynamic Constraint Checking For THE JVM
}

\author{
Konrad Grzanek \\ IT Institute, University of Social Sciences \\ 9 Sienkiewicza St., 90-113 Lodz, Poland \\ kgrzanek@spoleczna.pl
}

\begin{abstract}
Using formal methods for software verification slowly becomes a standard in the industry. Overall it is a good idea to integrate as many checks as possible with the programming language. This is a major cause of the apparent success of strong typing in software, either performed on the compile time or dynamically, on runtime. Unfortunately, only some of the properties of software may be expressed in the type system of event the most sophisticated programming languages. Many of them must be performed dynamically. This paper presents a flexible library for the dynamically typed, functional programming language running in the JVM environment. This library offers its users a close to zero run-time overhead and strong mathematical background in category theory.
\end{abstract}

Keywords: Formal software verification, software quality, dynamic type-checking, functional programming, category theory, Clojure

\section{Introduction}

Despite an apparent progress in programming languages theory and practice, the IT industry still experiences problems achieving a desired level of software quality and reliability. M. Thomas [2] mentions that there are from 4 up to 50 bugs (on average) in every 1 thousand lines of production code. This is why the computers are still problematic to rely on in the (not only) safety and mission critical areas of life [1], and the non-critical software is usually hard to use, has got a lowered level of security due to hidden bugs that may even not exhibit themselves on a regular usage basis, but may be exploited by the ones who search for vulnerabilities with an intention to steal information or to introduce other kinds of costly confusion.

This paper is a result of some real-life, production-related experiences and following considerations regarding when and how to perform constraints checks and other kinds 
of formal software verification in a dynamically-typed programming language for the Java Virtual Machine (JVM) environment. We argue that it is a reasonable decision to imply lots of these checks on the time of the program's execution. We also provide a library called $c h$ that allows to define and perform some run-time constraint checks. This article may be treated as a good introduction to how this library is implemented and how it can (and should) be used.

\subsection{Reasoning About Software Correctness}

Scale of contemporary software systems together with the fact that it is intended to run in a multi-tasking environment makes the formal verification methods a strong requirement not an option now. Growing popularity of tools like TLA+ (L. Lamport [5]), temporal logic, and even the hand proofs in software creation process confirm the growing need to become more and more dependent on the beauty of mathematical verification of software systems.

It is a commonly accepted truth that type systems in programming languages are a very strong point in the formal verification of software. Advances in type theory and practice $[3,4]$ have led to development of programming languages that are particularly effective in catching a variety of common bugs. Let us mention Haskell [9] or Ada here. Lamport [6] says:

„Types have become ubiquitous in computer science. [...] Types do more good than harm in a programming language: they let the compiler catch errors that would otherwise be found only after hours of debugging."

Strong typing means that the expressions in a language contain no implicit data conversions (coercions) that could lead to an unintentional loss of information. Static type system is the one in which the compiler (more generally speaking - a static verification tool, the one that reads and analyzes source code) is responsible for making a proof (or dis-proof) that a computer program does not violate any of the type-related invariants that are amenable to pre-run analysis. On the other side, the dynamically typed languages are these that leave at least some of the verifications of invariants for the run-time. We should also be aware that there are type-based invariants that may be verified neither statically, nor dynamically. For example, it is impossible to prove the correctness of a famous quick-sort algorithm using a type checker of any kind (for a discussion see [22]). In such cases using a formal method like the TLA+ ahead of the implementation phase alone to prove correctness, or even providing a hand proof is priceless. In any other scenario relying on the type system is a good idea. Typing the specification languages is a completely different problem - for more on that subject, please see [6].

\subsection{Discussion on Static and Dynamic Type-Checking}

Using a programming language with a static type checker seems a robust method of eliminating bugs in software. When considering only the type-related aspects of a language this statement leads to an immediate conclusion that the dynamic type checking should be avoided at all cost. But the language is much more that that, and there are 
multiple reasons why the dynamically typed programming languages have made such a great success in the industry. Among the others, the dynamic languages:

- Allow to perform immediate tests of the functionality being implemented by using REPL (Read-Eval-Print Loop).

- In the case of languages from the Lisp family, like Clojure [7, 8] it is even possible to compile new functionality without stopping the running program. The REPL execution takes part in the same environment in which the production software runs, we have no debug-release cycle here, and that feature alone is a great productivity booster.

- A lack of static type system allows to define programming constructs that are intended to be run in contexts that would be very hard or even impossible to describe using a set of static type-related constraints. Some Lisp macros are good example here.

Even when we decide to rely on a static type checker we should be aware that the extent to which we will be able to verify programs using this tool is limited to what can be expressed in the rules of the type system, and this extent has bounds. Other checks/verifications/proofs must be performed anyway either on the run-time or by hand proving. Static type systems generally verify that the elements of a system fit together as a structure, and only sometimes can prove or disprove their homeostasis and well-functioning over a period of time.

\subsection{Functional Programming}

Another means of establishing high level of software quality and reliability is using functional programming languages like Haskell, Clojure, Erlang. The impact of the immutability of data structures on software correctness, its predictability and a relative ease of searching for bugs may be at least as big as using the type checker to find the mistakes statically. Out of the mentioned three languages two are dynamically typed (Erlang, Clojure) and they are highly successful even in mission critical domains.

\subsection{Existing Solutions for Clojure}

Clojure programming language $[7,8]$ is one of the most interesting contemporary JVMtargeted languages. It belongs to Lisp family, and - as its elders - is dynamically typed. This section presents current approaches that exist in this language, and that are related to the problems formulated above.

One attempt to employ some kind of static type checking is clojure/core.typed library [20]. This solution uses type annotations and a static type checker. Unfortunately, the realization has severe performance problems as described in the discussion [21]. Moreover, due to the reasons described in the paragraphs above a full static type checker does not make a perfect fit with respect to the pragmatics of using Lisps and to the overall idea of implementing software quickly.

Another similar library that suffers similar problems is prismatic/schema [19]. In the case of this solution, we find it better in terms of the overall usability and performance, but the type annotations a kind of get in the way with the normal ways of using Lisp, that is - writing as much as possible using s-expressions. 
Finally, the most promising project in this domain is Cognitect's clojure.spec [17, 18]. It may become a de-facto standard specification tool, but its goals are slightly different than the ones we are looking at. Its development process is in its early stages, as the adoption in the industry.

These considerations led us to the idea of creating a custom solution - Clojure library meeting the requirements of dynamic specification/type/invariants validator, with the following assumptions:

- being deeply rooted in functional programming [9] and using notions from the category theory [10],

- consistency with the Lisp nature of Clojure programming language, by using sexpressions only (Lisp as a „,big ball of mud”),

- relying on fast dynamic type-checking routines of the JVM - the *instanceof* operator,

Our solution is called kongra/ch [12,13], shortly ch, and it has been successfully used in kongra/prelude $[14,15]$ and aptell $[16]$ projects. The following sections of the paper are detailed description of its implementation and possible use.

\section{Essentials of the ch Library}

Every predicate check uses the following procedure to generate a message describing the value, together with its type, that violates the check:

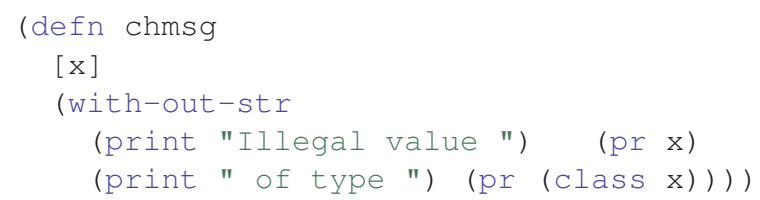

When executed in the REPL the procedure works as follows:

user> (chmsg 123)
"Illegal value 123 of type java.lang.Long"

or, for nil values:

user> (chmsg nil)

"Illegal value nil of type nil"

The most essential syntactic structure in the ch library is a $(c h .$.$) form. Due to the$ implementation issues the form is intended to be used both as an assertion and as a boolean-valued function. The definition begins with a supporting function pred-callform:

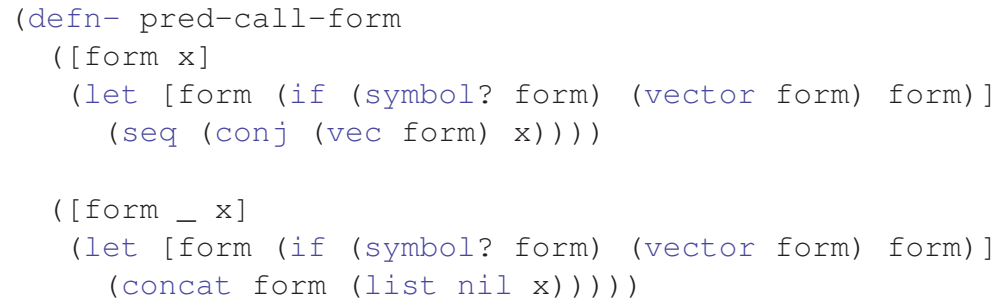


and the actual $c h$ macro that uses the function to generate a target s-expression, either an assertion or a predicate-like call:

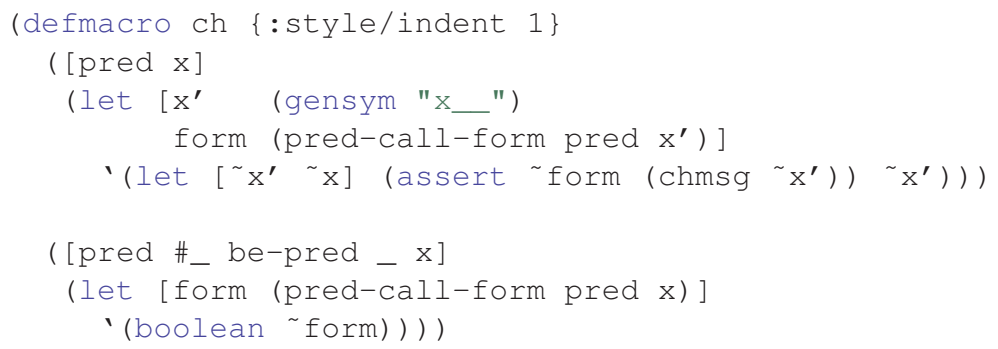

To see, what actually happens when a compiler encounters the (ch...) form, please take a look at the expression (ch nil? 123). This expression evaluates the function nil? belonging to the Clojure standard library against the argument, integral (Long) value 123. The target form is

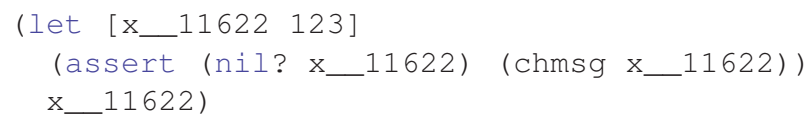

The compiler introduces an additional local variable $\mathrm{x}_{--1} 11622$ that holds the value of an evaluated 123 input value (expression from compiler's point of view) and executes the (assert...) on it using the passed nil? function an assert's predicate. After a successful evaluation the evaluated value of $\mathrm{x}_{-1} 11622$ is returned resulting in the desired behavior. This time it's failure, because 123 is not a nil value:

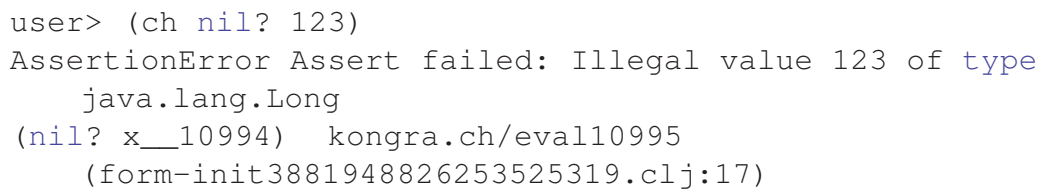

If we decide to use (ch...) using its predicate "nature", like (ch nil? :as-pred 123), we get:

(boolean (nil? 123))

and the evaluation of the form ends with false value being returned.

Now, let's talk about the performance. All the following performance benchmarks were taken in the commodity hardware environment: Intel i7-5500U, 16 GB of RAM, Ubuntu 14.04 64-bit using the awesome criterium $^{1}$ library for benchmarking Clojure codes. Additionally we have: CIDER 0.14.0 (Berlin), nREPL 0.2.12, Clojure 1.8.0, Java 1.8.0_121. At first a simple expression:

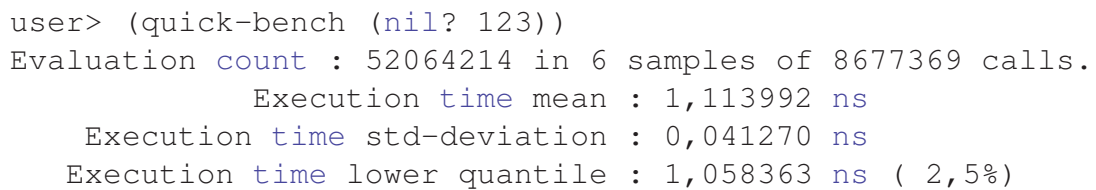

${ }^{1}$ https://github.com/hugoduncan/criterium 


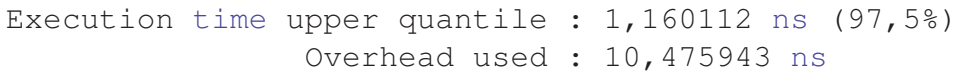

and the corresponding predicate form of $(c h \ldots)$ :

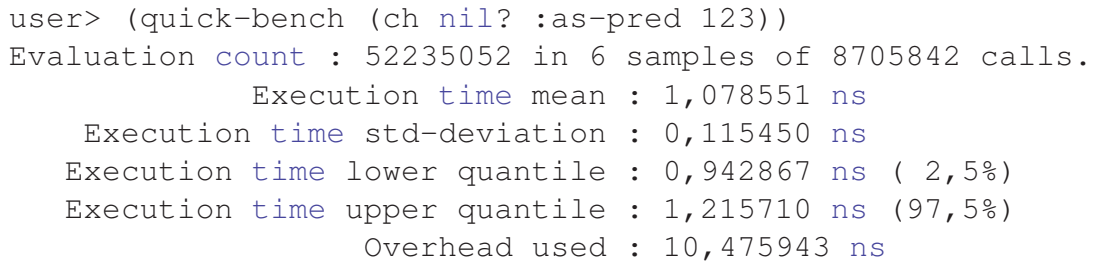

Also for the assertion:

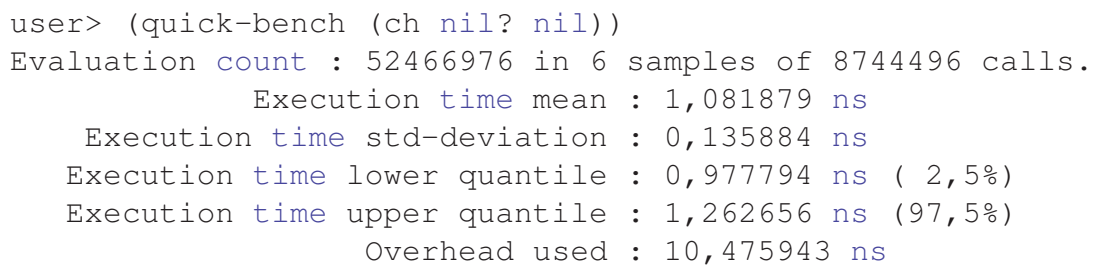

We can clearly see that there is no apparent overhead of the call. This is only an introductory example, so it is impossible to reason now about the target performance loss when applying this approach to a production software. This will be discussed in further parts of the paper.

\section{Generator of $\mathrm{Ch}(\mathrm{eck}) \mathrm{s}$}

The library would be far from being useful, if the user would be forced to use raw $(c h . .$.$) forms everywhere. Instead, we introduce the (d e f c h . .$.$) macro that allows the$ programmer to define his own named checks. The macro code goes as follows:

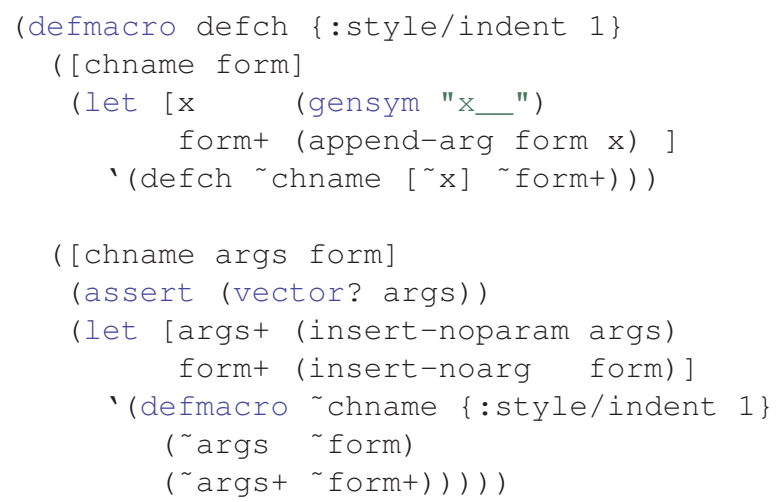

To do its job, the macro performs some manipulations with the arguments and the shape of the target form, which is a subsequent macro in this case. So we may say that $d e f c h$ is a macro-writing macro. 
The arguments must be enhanced do support a non-assertion (predicate) use, because - as in the case of raw ch - we tend to operate both in assertion and in predicate mode. The arguments of the target macro are prepared using the following insertnoparam:

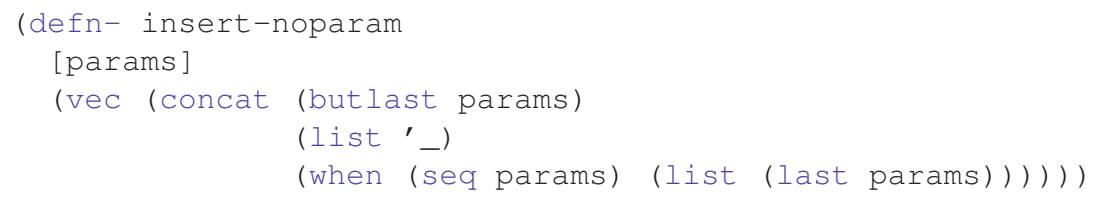

while the two following procedures prepare the predicate target form:

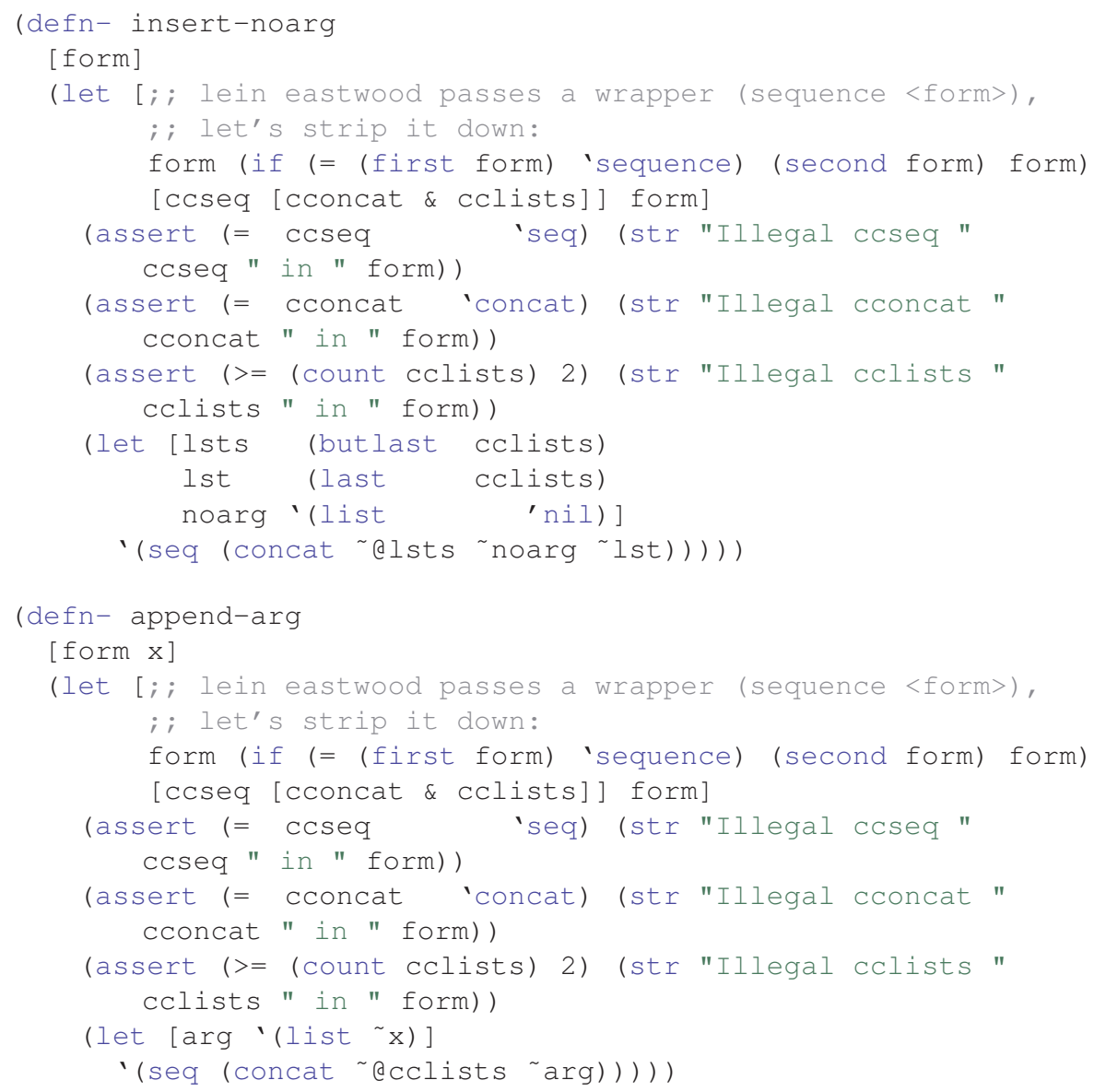

Finally we may take a look at how this entirety works together.

\section{Unit Type $\mathrm{Ch}(\mathrm{eck})$}

Unit type is a very useful type in many programming languages. Unit type has exactly one value. It is so called terminal object in category of types and typed functions. In 
Execution time upper quantile : 7,444272 ns $(97,5 \%)$

Overhead used : 10,475943 ns

To look more deeply in what happens under the hood in these test procedures, let's use no.disassemble ${ }^{2}$ library to view the resulting byte-code for foo:

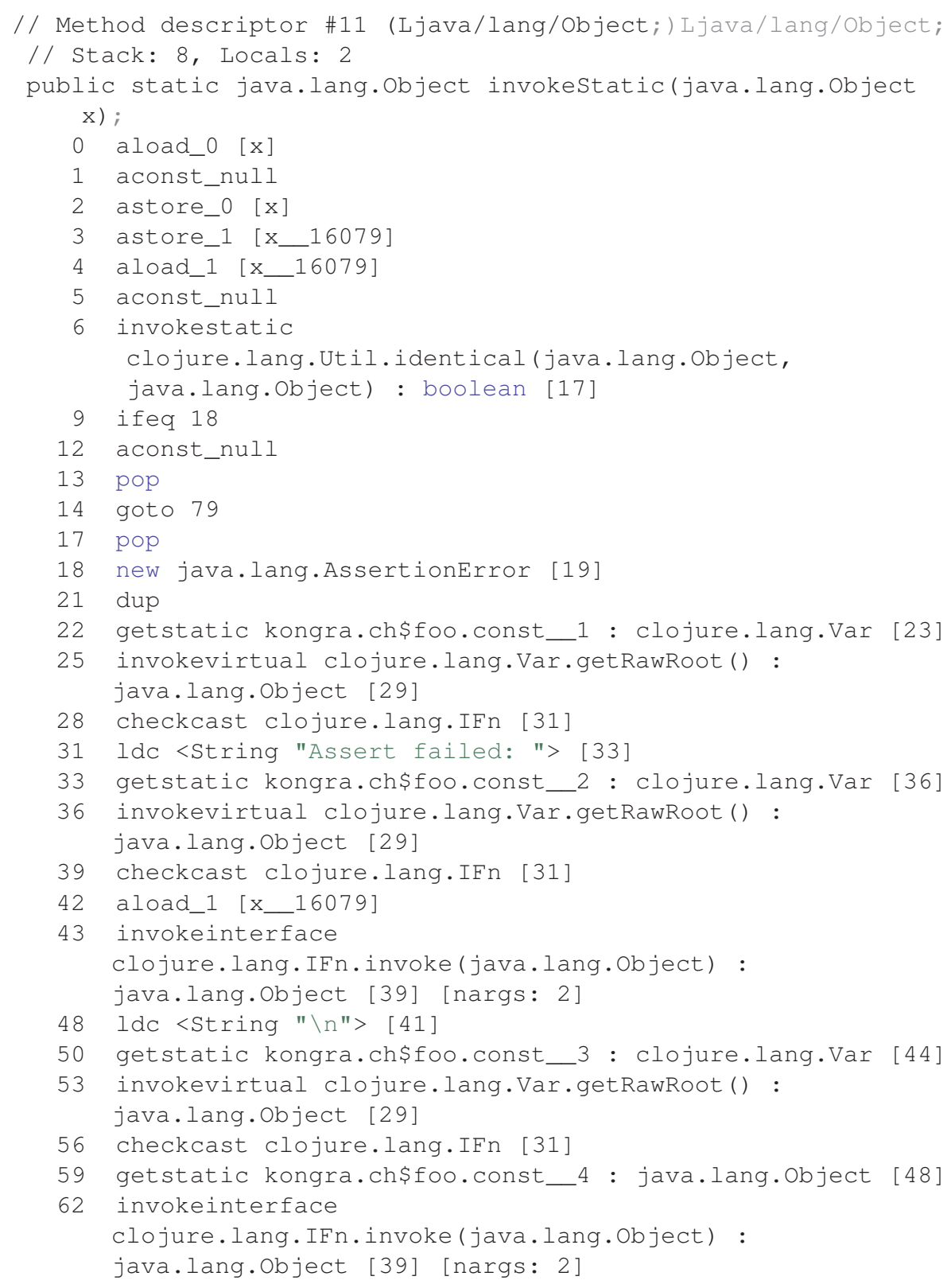

${ }^{2}$ https://github.com/gtrak/no.disassemble 
67 invokeinterface

clojure.lang.IFn.invoke (java.lang.object,

java.lang.object, java.lang.object, java.lang.object) :

java.lang.object [51] [nargs: 5]

72 invokespecial

java.lang.AssertionError(java.lang.object) [54]

75 checkcast java.lang.Throwable [56]

78 athrow

79 aload_1 [x_16079]

80 aconst_null

81 astore_1

82 areturn

When Clojure direct linking is enabled, we have an even more optimized code like:

public static java.lang.Object invokestatic(java.lang.object

$\mathrm{x})$;

aload_o [x]

aconst_null

astore_0 $[\mathrm{x}]$

astore_1 [x_12541]

aload_1 [x_12541]

aconst_null

invokestatic

clojure.lang.util.identical (java.lang. Object,

java.lang.object) : boolean [17]

9 ifeq 19

12 getstatic java.lang.Boolean.FALSE : java.lang.Boolean [23]

15 goto 22

18 pop

19 getstatic java.lang.Boolean.TRUE : java.lang.Boolean [26]

22 dup

23 ifnull 37

26 getstatic java.lang.Boolean.FALSE : java.lang.Boolean [23]

29 if_acmpeq 38

32 aconst_null

33 pop

34 goto 92

37 pop

38 new java.lang.AssertionError [28]

41 dup

42 ldc <String "Assert failed: "> [30]

44 iconst_3

45 anewarray java.lang.object [32]

48 dup

49 iconst_0

50 aload_1 [x_12541]

51 invokestatic 
Grzanek K.

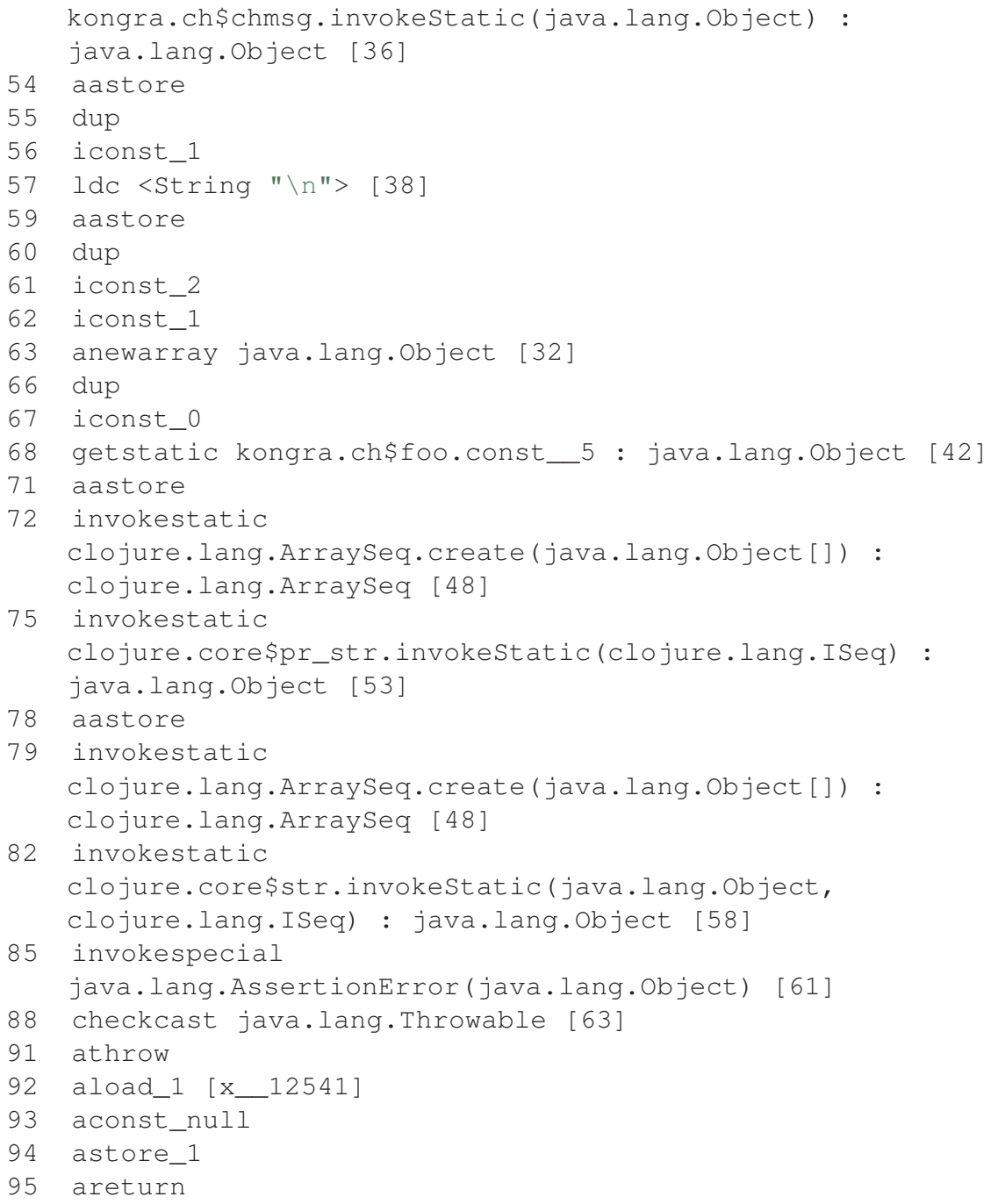

The code is actually a call to the (assert ...) form as defined in the original (ch ...) mechanism. With this in mind we may be certain to get a very efficient checkinstrumenting code in all the places we use the ch library, and the actual cost of every check depends solely on the nature (and cost) of the predicates he uses. It is the responsibility of the programmer to keep them as cheap as possible. From what is well known the instanceof predicates in the JVM are particularly fast and cheap both on the CPU and the memory side. The following section discusses a way the ch library approaches the type-checks. 


\section{Class Membership Ch(eck)s}

To come up with a proper, fast, run-time type checking, we first provide the following macro that makes the instance? call:

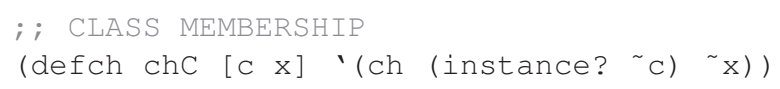

Then we are ready to define another utility macro-writing macro $d e f c h C$ that allows the programmer to define his own type checks (besides the ones defined in the ch library, see Appendix A):

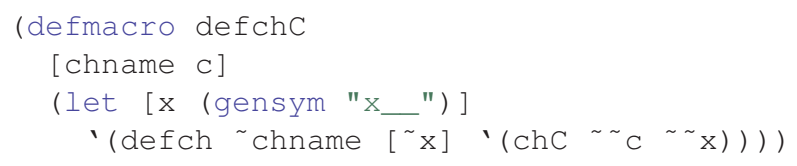

Among the others the check for java.lang.Long type is defined like: (defchC chLong Long). Now we can turn the procedure (defn foo $[x] x$ ) into (defn foo $[x]$ (chLong $x)$ ). The resulting procedure has the performance profile as in the following benchmarking result:

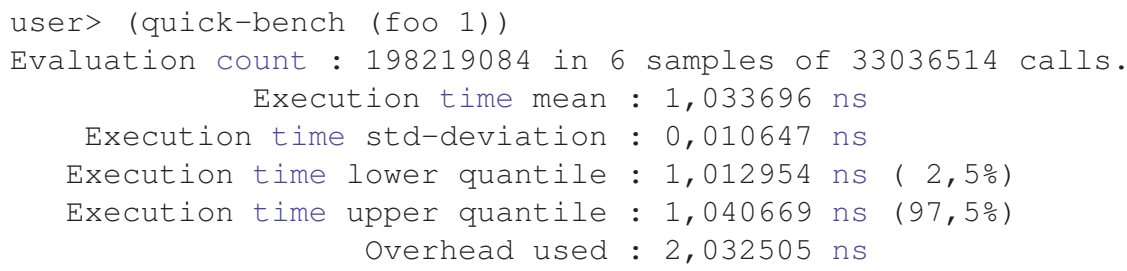

Similarly for java.lang.String we have:

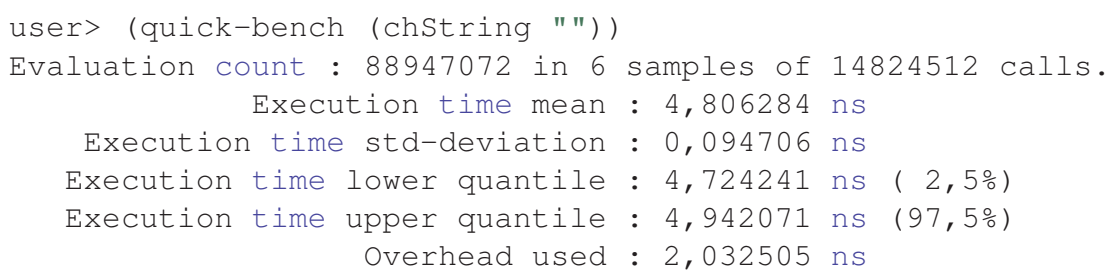

These benchmarks shows two things:

- The modern JVM has perfect ways to optimize type checks up to a level that is almost hard to notice from a point of a programmer who writes common code, even in the performance-critical parts.

- Our approach makes no overhead when calling these mechanisms and making the JVM actually do its job.

\section{Object Type Equality $\mathrm{Ch}(\mathrm{eck}) \mathrm{s}$}

In a common programmers' practice we often have to ensure two objects have exactly the same type. Check chLike, as defined below, serves exactly that: 


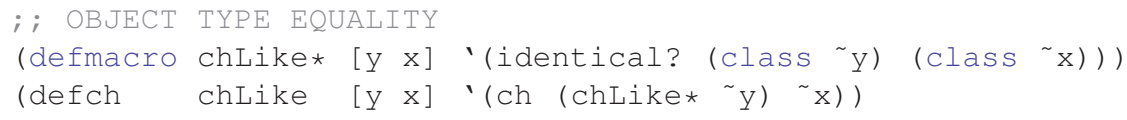

Let's take look at its performance benchmark, here for two Strings:

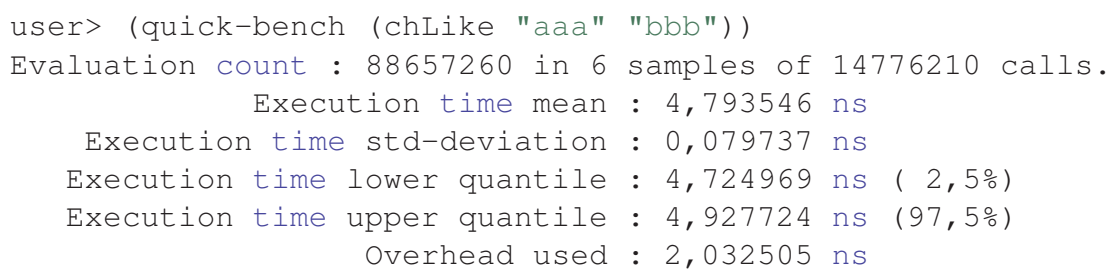

\section{Product (Pair/Tuple) and Co-Product (Discriminated Union Type) $\operatorname{Ch}($ eck)s}

To apply more compound checks that use logical operators we define the following generator of predicate checks:

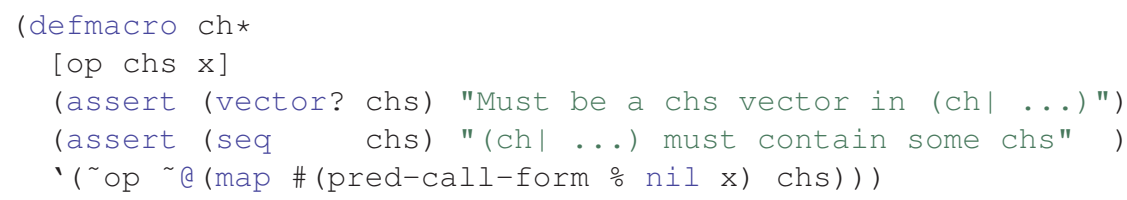

The generator is used to define checks for tuple types and for discriminated union types:

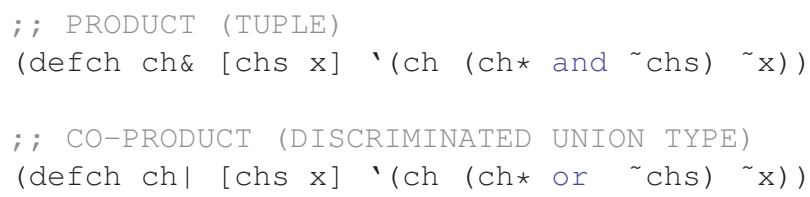

A special case here is a co-product of exactly two types, known as Either $a$ b type constructor in some languages (e.g. Haskell), and its variant - the Maybe a type constructor, that can be defined as typeMaybea $=$ Either () $a$. The ch library specifies them as follows:

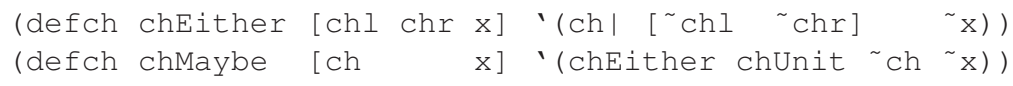

And an example benchmark:

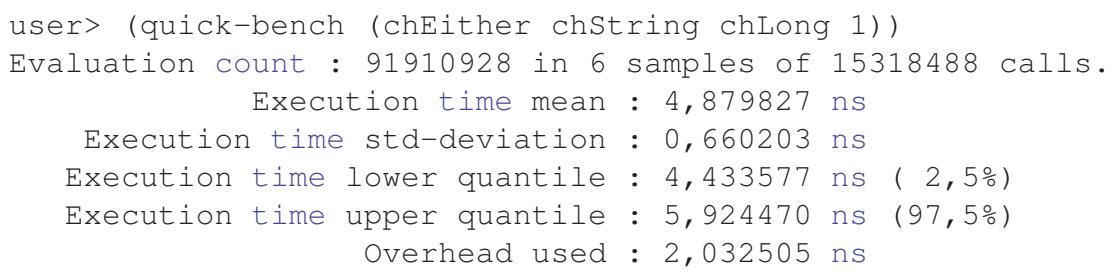


Again, we cannot see any significant impact on the performance, other than few nanoseconds.

\section{Registry of Ch(eck)s}

Additionally the ch library provides a registry of checks, that helps the programmer to understand, what kind of checks an object or a collection of objects fulfill. The registry is actually a mapping from string (a name) into a check:

(def ^:private CHS (atom \{\}))

To register a new check we use the following macro:

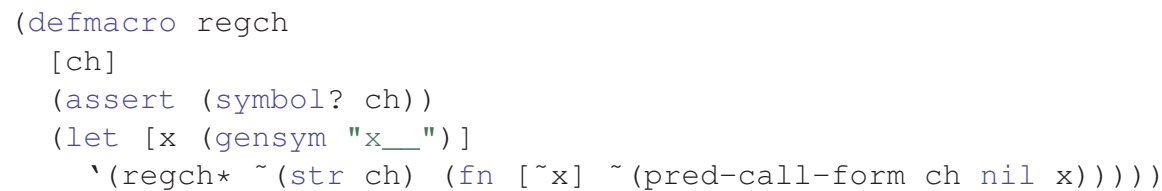

together with its back-end:

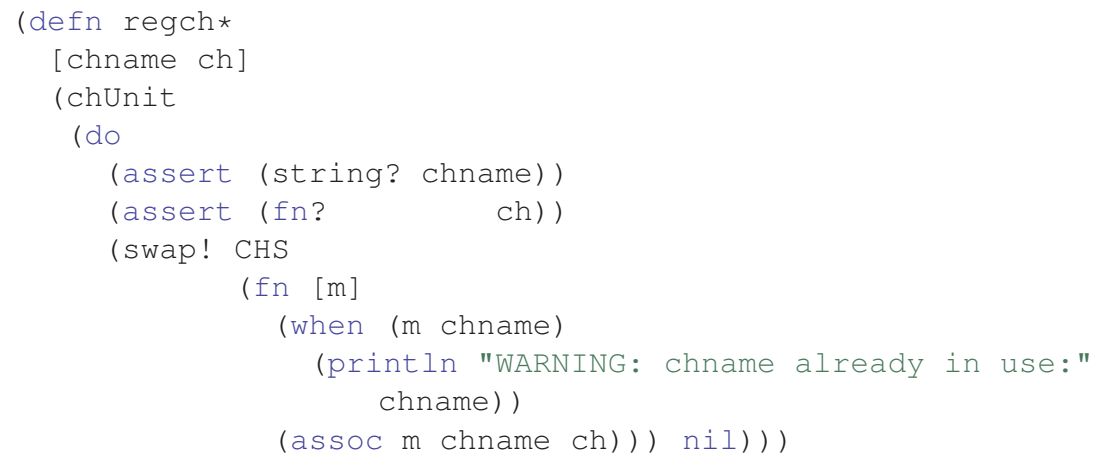

Now we may use chs function:

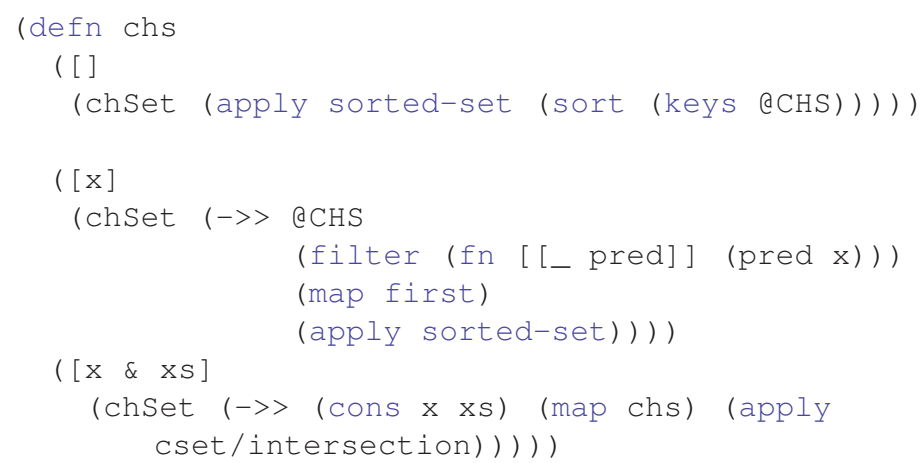

to reach for the information, e.g.:

user> (chs 1)

\#\{"chInteger" "chLong" "chNumber" "chRational"\} 
Grzanek K.

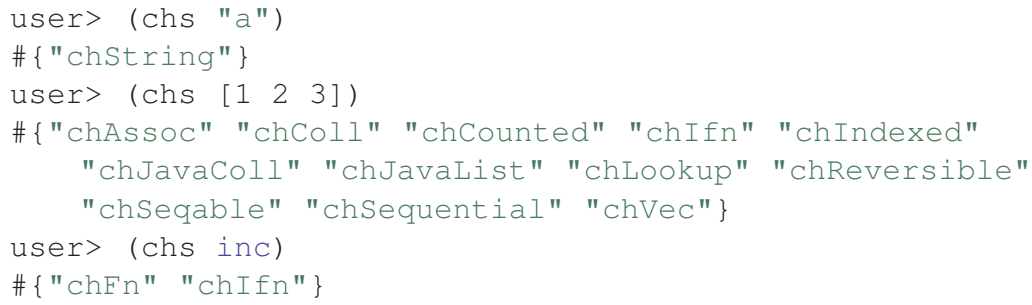

There is also a possibility to ask for checks common for a set of objects:

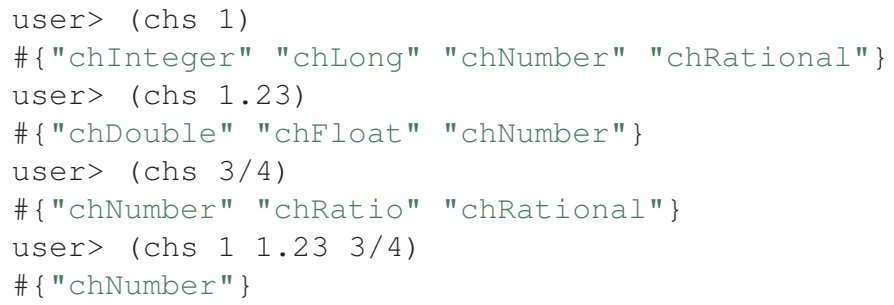

Using the information provided the programmer can make a decision on what checks to use in a particular situation.

\section{Example Use in Production Setting}

The ch library is used extensively in production. Among the others it was used to tag some of the elements of kongra/prelude package. With the following namespace declaration:

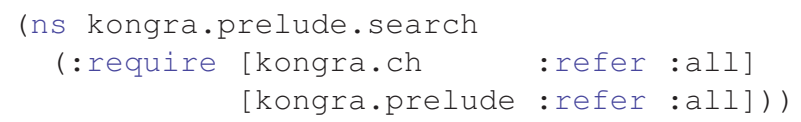

we define tree-search routines in the kongra.prelude.search namespace. In the first place we define a combiner function that controls the tree search process, by performing order-wise concatenation of search space elements. The concatenation operates on sequences and returns a sequence, thus the use of $c h S e q$ in the following code:

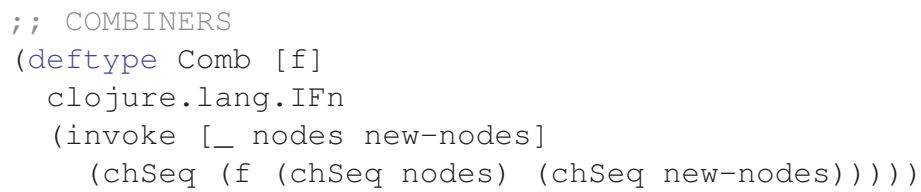

For the combiner we define a chComb ch(eck) and a proper constructor (consComb):

(defchC chComb Comb)

(defn conscomb [f] (Comb. (chIfn f)) )

The combiners for breath-first and depth-first search strategies are defined as follows:

$\begin{array}{llr}\text { (def breadth-first-combiner } & \text { (consComb concat)) } \\ \text { (def lazy-breadth-first-combiner } & \text { (conscomb lazy-cat')) }\end{array}$ 


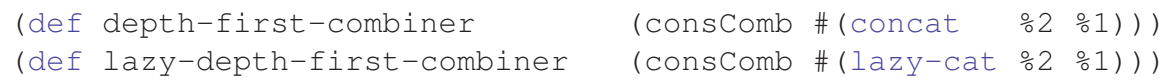

Using exactly the same pattern we define an abstraction for goal functions

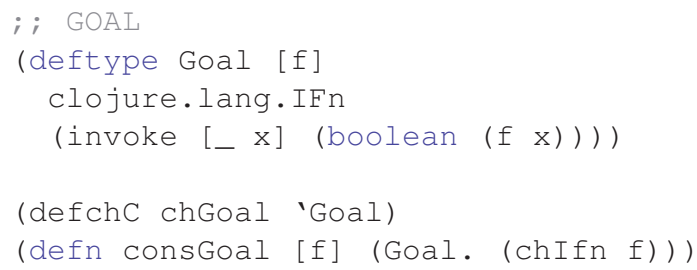

and for adjacency generators for the tree structure:

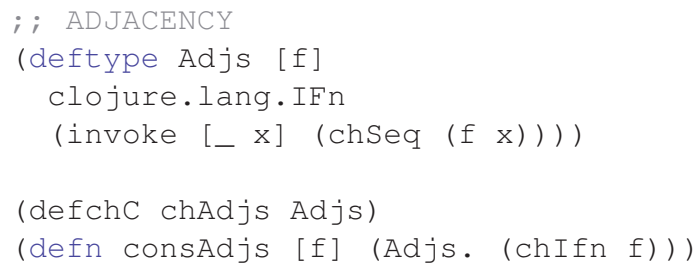

Finally the general tree-search procedure uses all the checks defined earlier as presented in the following listing:

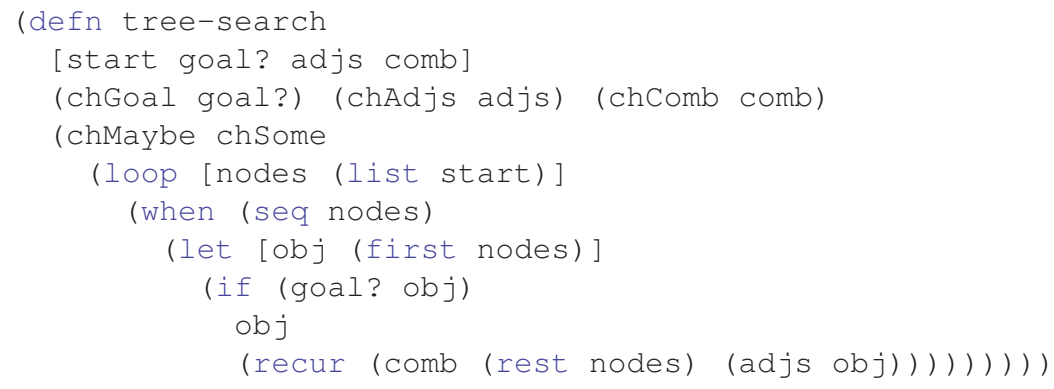

Both major search strategies have the implementations like:

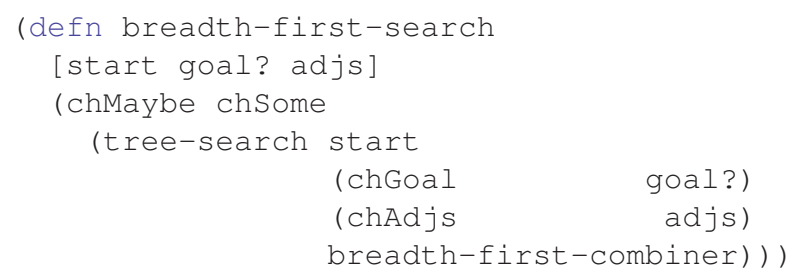

and:

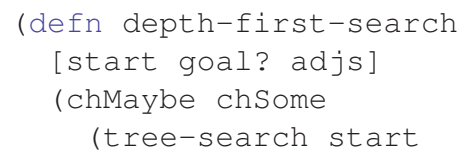


Grzanek K.

$\begin{array}{cc}\text { (chGoal } & \text { goal?) } \\ \text { (chAdjs } & \text { adjs) } \\ \text { depth-first-combiner)) }\end{array}$

A very useful procedure breadth-first-tree-levels that returns consecutive depth levels of a tree also uses the mechanisms.

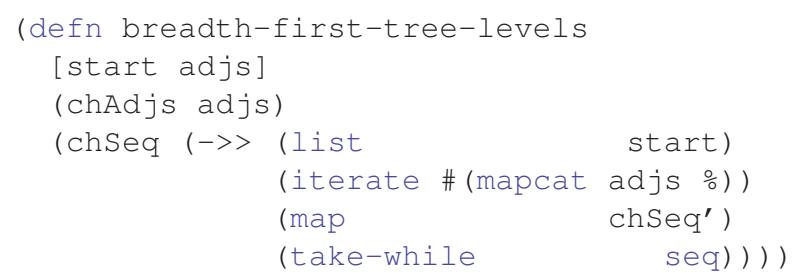

And finally the following traversal mechanism returns a lazily evaluated sequence of all tree nodes visited according to a breadth-first strategy:

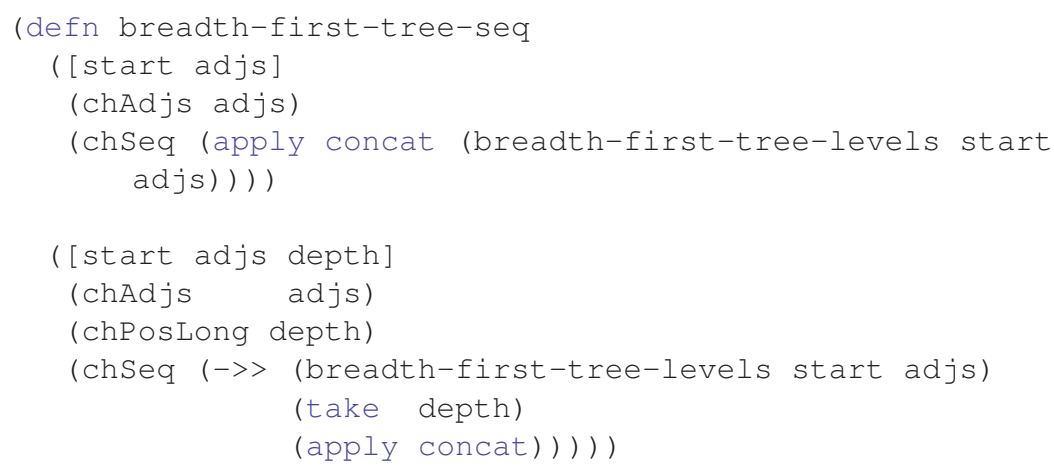

\section{Plans for the Future Development}

Performing the dynamic (run-time) checks for various constraints, including the verification of types is not enough to ensure proper integrity of software projects. Many factors come to mind here, including:

- Multiple versions of libraries that software projects depend upon, together with the information on the actual use of these dependencies, circularity of dependencies, and general lack of reliable sources of coherent packages (libraries) bundled together.

- Lack of the ability to effectively model highly complex systems, like the Java 8 Language Specification, being turned on into a working compiler or at least a static analyzer working according to the rules present in the specification. The estimated amount of work for creating Java compiler is hundreds of man-years, while it would be great to be able to perform activities like that in time an order of magnitude shorter. Problems of this kind were already discussed by us in [11].

These issues and the possibility to solve them in an uniform way will be subjects of our further research activities. We tend to make $c h$ library a part of the solution. 


\section{References}

1. Thomas M., 2016, How Can Software Be So Hard?, Gresham College Lecture, Feb. 2016, https://www.youtube.com/watch?v=VfRVz1iqgKU

2. Thomas M., 2015, Should We Trust Computers, Gresham College Lecture, Oct. 2015, https://www.youtube.com/watch?v=8SZfjvlbpMw

3. Pierce B.C., 2002, Types and Programming Languages, 1st Edition, MIT Press, ISBN-10: 0262162091, ISBN-13: 978-0262162098

4. Pierce B.C., 2004, Advanced Topics in Types and Programming Languages, MIT Press, ISBN-10: 0262162288, ISBN-13: 978-0262162289

5. Lamport L., 2002, Specifying Systems, The TLA+Language and Tools for Hardware and Software Engineers, Addison Wesley, ISBN: 0-321-14306-X

6. Lamport L., Paulson L.L., 1999, Should Your Specification Language Be Typed?, ACM Transactions on Programming Languages and Systems, Vol. 21, No. 3, pp. $502-526$

7. Emerick Ch., Carper B., Grand Ch., 2012, Clojure Programming, O’Reilly Media Inc., ISBN: 978-1-449-39470-7

8. Fogus M., Houser Ch., 2014, The Joy of Clojure, Manning Publications; 2 edition, ISBN-10: 1617291412, ISBN-13: 978-1617291418

9. Bird R., Wadler R., 1988, Introduction to Functional Programming. Series in Computer Science (Editor: C.A.R. Hoare), Prentice Hall International (UK) Ltd

10. Awodey S., 2010, Category Theory, Second Edition, Oxford University Press

11. Grzanek K., 2012, Prerequisites for Effective Requirements Management, Journal of Applied Computer Science Methods, Vol. 4, No 1, pp. 21-28

12. Grzanek K., 2017, ch GitHub Repository, https://github.com/kongra/ch

13. Grzanek K., 2017, ch Clojars Page, https://clojars.org/kongra/ch

14. Grzanek K., 2017, prelude GitHub Repository, https://github.com/kongra/prelude

15. Grzanek K., 2017, prelude Clojars Page, https://clojars.org/kongra/prelude

16. Grzanek K., 2017, aptell GitHub Repository, https://github.com/kongra/aptell

17. Clojure Team, 2017, clojure.spec Rationale, https://clojure.org/about/spec

18. Clojure Team, 2017, clojure.spec Guide, https://clojure.org/guides/spec

19. Plumatic, 2017, prismatic/schema GitHub Repository, https://github.com/ plumatic/schema 
20. Clojure Team, 2017, clojure/core.typed GitHub Repository, https://github.com/ clojure/core.typed

21. CircleCI, 2015, Why we're no longer using core.typed, https://circleci.com/blog/ why-were-no-longer-using-core-typed/

22. Hacker News, 2015, Why were no longer using core.typed - discussion, https:// news.ycombinator.com/item?id=10271149

\section{A Appendix: Selected Pre-defined Checks}

In the beginning we define the following type checks for basic types (classes) belonging to the standard Clojure library and/or forming the Clojure run-time environment:

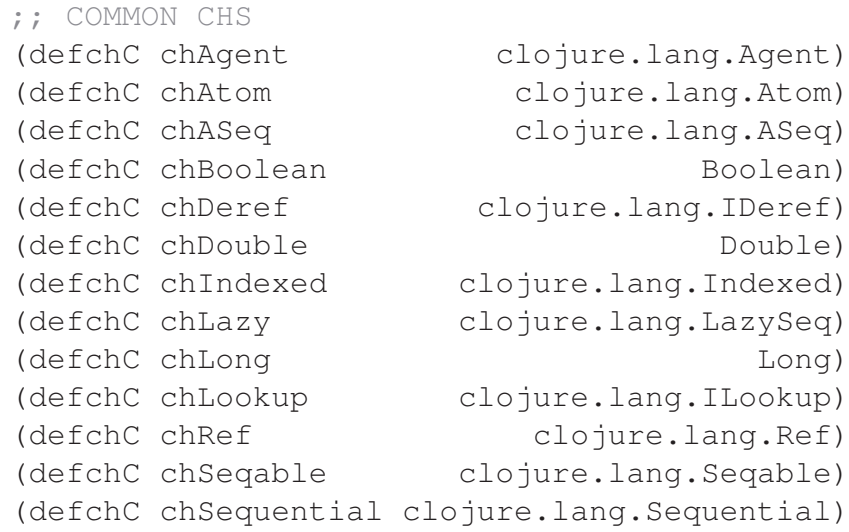

Another family of the basic checks is the checks defined upon some essential predicates belonging to the standard library of the language:

$\begin{array}{ll}\text { (defch chAssoc } & \text { (ch associative?)) } \\ \text { (defch chChar } & \text { '(ch char?)) } \\ \text { (defch chclass } & \text { (ch class?)) } \\ \text { (defch chcoll } & \text { (ch coll?)) } \\ \text { (defch chCounted } & \text { (ch counted?)) } \\ \text { (defch chDecimal } & \text { (ch decimal?)) } \\ \text { (defch chDelay } & \text { '(ch delay?)) } \\ \text { (defch chFloat } & \text { (ch float?)) } \\ \text { (defch chFn } & \text { '(ch fn?)) } \\ \text { (defch chFuture } & \text { (ch future?)) } \\ \text { (defch chIfn } & \text { '(ch ifn?)) } \\ \text { (defch chInteger } & \text { (ch integer?)) } \\ \text { (defch chKeyword } & \text { (ch keyword?)) } \\ \text { (defch chlist } & \text { '(ch list?)) } \\ \text { (defch chMap } & \text { '(ch map?)) } \\ \text { (defch chNumber } & \text { '(ch number?)) } \\ \text { (defch chRatio } & \text { '(ch ratio?)) } \\ \text { (defch chRational } & \text { (ch rational?)) }\end{array}$


Low-Cost Dynamic Constraint ...

$\begin{array}{llr}\text { (defch } & \text { chRecord } & \text { '(ch record?)) } \\ \text { (defch chReduced } & \text { '(ch reduced?)) } \\ \text { (defch } & \text { chReversible } & \text { (ch reversible?)) } \\ \text { (defch chSeq } & \text { '(ch seq?)) } \\ \text { (defch chSorted } & \text { '(ch sorted?)) } \\ \text { (defch chString } & \text { (ch string?)) } \\ \text { (defch chSymol } & \text { (ch symol?)) } \\ \text { (defch chVar chVec } & \text { '(ch var?)) }\end{array}$

Finally we introduce type checks for few basic Java collection interfaces. It is worth mentioning, that all Clojure collections implement one of these interfaces:

$\begin{array}{rr}\text { (defchC chJavaColl } & \text { java.util.Collection) } \\ \text { (defchC chJavalist } & \text { java.util.List) } \\ \text { (defchC chJavaMap } & \text { java.util.Map) } \\ \text { (defchC chJavaset } & \text { java.util.Set) }\end{array}$

\section{B Appendix: Selected Tests}

The ch library is covered with unit tests. Here we present few of them to give the reader another opportunity to get familiar with syntax and behavior of the library. In the following codes we use the namespace definition as below:

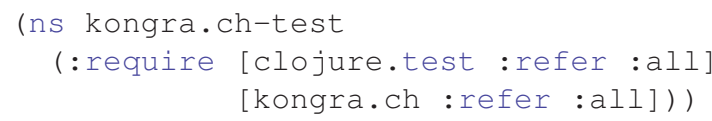

First of all let's define few types with their accompanying type checks:

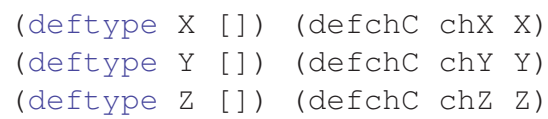

We also define the following simple checks of various kinds:

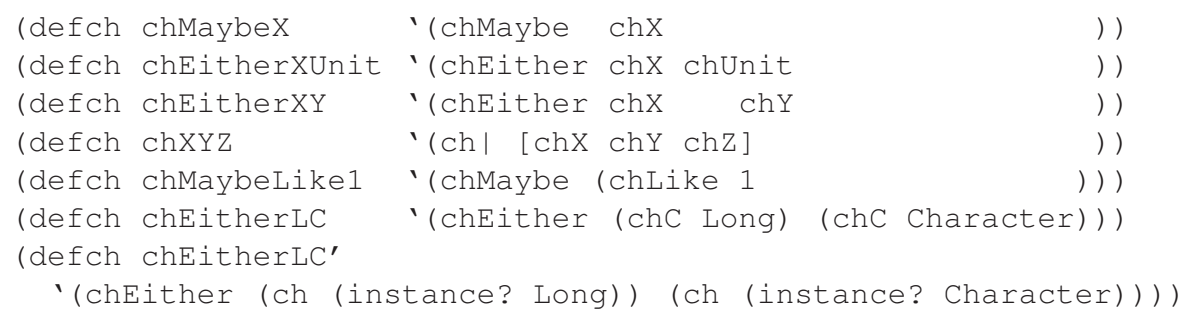

as well as the compound one:

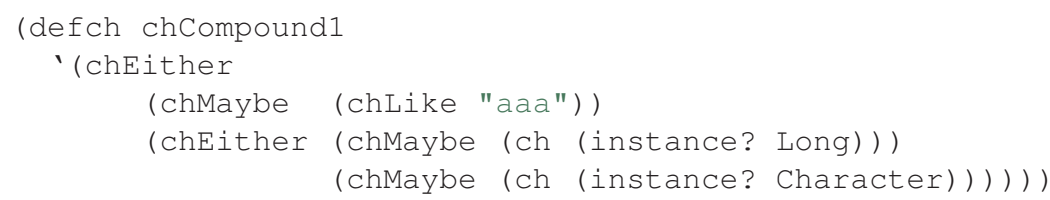


Here the test cases follow:

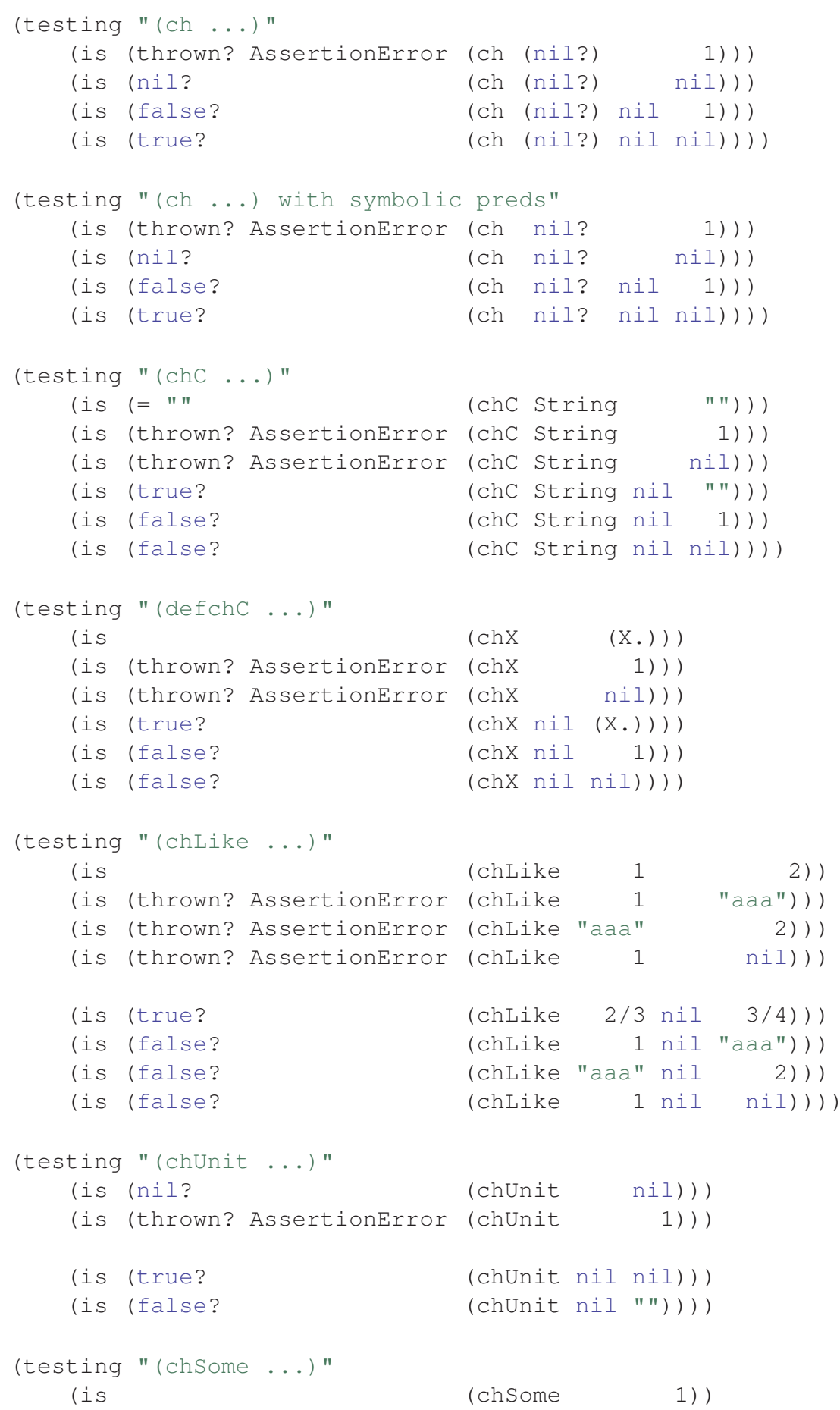




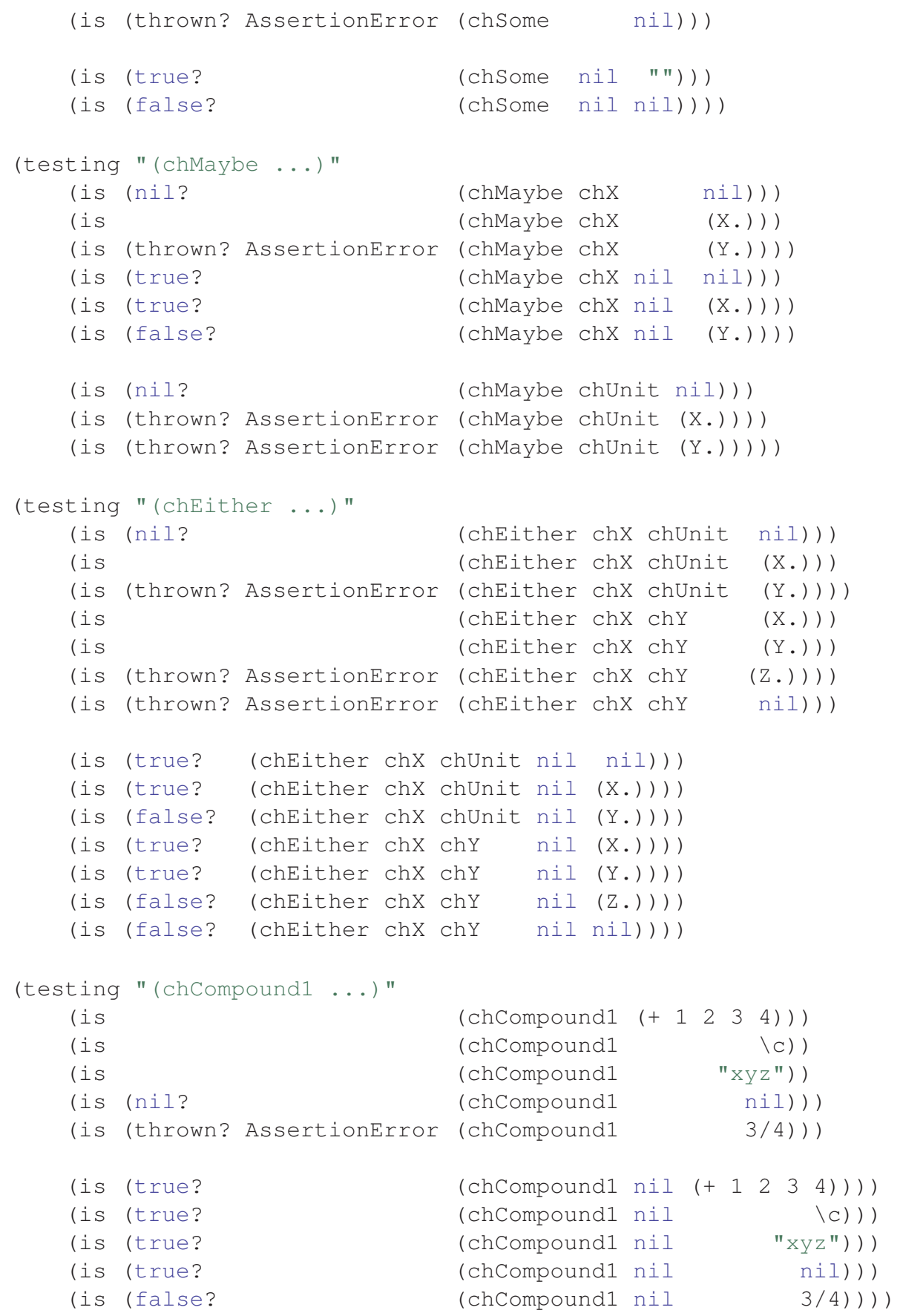

